\title{
Una cierta idea de la filosofía: acerca de la recepción y no recepción de Nicolás Gómez Dávila en Francia
}

Michäel Rabier

LIPHA Paris-Est, Francia 


\title{
Una cierta idea de la filosofía: acerca de la recepción y no recepción de Nicolás Gómez Dávila en Francia*
}

Resumen: la recepción de la obra de Nicolás Gómez Dávila en Francia ha sido muy escasa hasta el momento, sobre todo en el medio universitario. Este problema de la no recepción -más que de la recepción- está ligado no solamente y directamente a una cuestión editorial y/o de traducción, sino a un asunto intelectual y académico que tiene, según nuestra tesis, mucho que ver con la concepción francesa y moderna de la filosofía y a lo que hemos llamado la "anti-filosofía" gomezdaviliana.

Palabras clave: filosofía, anti-filosofía, ideología, zetética.

\section{A certain idea of the philosophy. About the reception and non-reception of Nicolás Gómez Dávila in France}

\begin{abstract}
Nicolás Gómez Dávila in France has been very little until the moment, mainly in university means. This problem of the no reception -more than of the reception- is not bound only and directly to a publishing question and/or of translation, but to an intellectual and academic subject that it has, according to our thesis, much that to see with the French and modern conception of the philosophy and to which we have called the gomezdavilian "anti-philosophy".
\end{abstract}

Keywords: philosophy, anti-philosophy, ideology, zetetic.

Fecha de recepción: 25 de agosto de 2018

Fecha de aceptación: 16 de enero de 2019

Forma de cita (APA): Michaël-Rabier. (2019). Una cierta idea de la filosofía: acerca de la recepción y no recepción de Nicolás Gómez Dávila en Francia. Revista Filosofía UIS, 18(1), doi: http://dx.doi.org/10.18273/revfil.v18n1-2019009

Forma de cita (Harvard): Michaël-Rabier. (2019). Una cierta idea de la filosofía: acerca de la recepción y no recepción de Nicolás Gómez Dávila en Francia. Revista Filosofía UIS, 18(1), 189-206.

Rabier Michaël: francés. Doctor en Filosofía Práctica, investigador asociado en LIPHA Paris-Est, Francia.

Correo electrónico: michael.rabier@gmail.com

ORCID: https://orcid.org/0000-0002-2329-7295

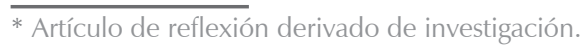




\section{Una cierta idea de la filosofía: acerca de la recepción y no recepción de Nicolás Gómez Dávila en Francia}

\section{La recepción de Nicolás Gómez Dávila en Francia}

Francia ha sido muy ingrata con Nicolás Gómez Dávila. Desafortunadamente, y paradójicamente, si se tiene en cuenta que, como él mismo lo confesó a Martin Mosebach: "Francia me formó". El país de Montaigne, su santo de predilección", ha sido fundamental en su formación intelectual, moldeando su forma mentis durante el periodo de juventud. En efecto, cabe recordar que Gómez Dávila vivió en París entre los 6 y los 23 años de edad (1919-1936) durante una época clave de su historia personal, y más aún de la historia francesa moderna: el entreguerras. Por lo tanto, sería muy interesante investigar la influencia de Francia en el pensamiento gomezdaviliano, y no solamente en la forma moralista y ensayística de su obra.

Pese a esto, en materia editorial, no existe en este momento una publicación completa disponible en francés de los escolios gomezdavilianos, y mucho menos obviamente de Textos I y Notas. Peor aún, la única traducción relativamente extensa, muy relativamente y muy criticable, de los escolios a cargo de la editorial Le Rocher está ahora agotada. Se trataba de escolios escogidos por Samuel Brussel, traducidos por Michel Bibard, en su colección "Anatolia" publicados respectivamente en 2003 y en 2005 bajo los títulos muy llamativos más no muy significativos de Les horreurs de la démocratie [Los horrores de la democracia] et Le réactionnaire authentique [El reaccionario auténtico]. Una selección del primero titulada Misère de la démocratie [Miseria de la democracia] fue publicada en el primer número de la revista hoy desaparecida Le Lecteur en 2003 (pp. 72-79).

Pero realmente no fueron estas las primeras traducciones al francés del autor bogotano. Debemos a Philippe Billé, primus inter pares, la publicación inaugural en Francia a cuenta de autor de una selección de algunos escolios de 1977, 1986 y 1992 en su Studia Daviliana. Études sur Nicolás Gómez Dávila, La Croix-

1 "Mis santos patrones: Montaigne y Burckhardt" (Gómez Dávila, 1977, I, 428). 
Comtesse (2003). Este librito que aparenta a un folleto incluye una bibliografía de las obras, traducciones y estudios sobre Nicolás Gómez Dávila, una presentación por Philippe Billé de los escolios, una traducción del artículo de Martin Mosebach "una visita a Nicolás Gómez Dávila", entrevistas a Alvaro Mutis y Juan Gustavo Cobo Borda y, cabe resaltar, un muy preciado índice onomástico de los cinco volúmenes de escolios aún todavía por completar y mejorar.

Billé descubrió Nicolás Gómez Dávila en 2000 por medio de Botho Strauss en su obra traducida al francés como Le Soulèvement contre le monde secondaire (1997) [La rebelión contra el mundo secundario], en alemán Aufstand gegen die sekundäre Welt, en la cual hace varias referencias al filósofo colombiano. Podemos así constatar que la primera recepción de Gómez Dávila en Francia pasa pues por Alemania, e incluso Italia dado el hecho que el mismo Samuel Brussel, como lo contó, descubrió al Bogotano leyendo por casualidad un artículo publicado en un periódico italiano. Incluso Billé resalta que las primeras citas en francés del autor colombiano se encuentran en este ensayo del dramaturgo alemán.

Mientras tanto había sido publicado en una modesta revista literaria francesa llamada Contrelittérature, No 14, verano 2004, una traducción del ensayo sobre el Reaccionario auténtico descubierto por el autor de estas líneas en la Revista Universidad de Antioquia con una presentación del pensador colombiano titulada: Nicolás Gómez Dávila, le scribe des siècles [el escriba de los siglos]. Algunos años más tarde siguió otra y última traducción de los escolios bajo el título muy cioranesco de Carnets d'un vaincu (2009) [cuadernos de un vencido] a cargo de Alexandra Templier, por la editorial L'Arche, en su colección "Tête-à-tête". Como se puede notar se trata de una traducción directa y literal de la edición austriaca [Aufzeichnungen des Besiegten. Fortgesetzte Scholien zu einem inbegriffenen Text. Karolinger, Wien, 1994] de los Sucesivos escolios a un texto implícito. Otra vez la recepción francesa de Gomez Dávila no hubiera sido posible sin la publicación en alemán de la editorial Karolinger.

Con esta escasa difusión en francés de la obra gomezdaviliana no se podía esperar una recepción muy amplía de su pensamiento, mucho menos en el ámbito universitario a excepción de la tesis de Francia Elena Goenaga (Trois moralistes: Marie Linage, François de la Rochefoucauld et Nicolás Gómez Dávila) dedicada por parte al pensamiento reaccionario del colombiano y sustentada en la universidad de Paris VIII en 2006, y nuestra investigación comparativa (Philosophie, gnose et modernité. Nicolás Gómez Dávila lecteur d'Eric Voegelin), diez años después, en mayor medida consagrada a la vida y obra como lector y pensador de Gómez Dávila presentada en la universidad de Paris XII. Sin embargo, hay que subrayar el interés reciente y esperanzador de algunas figuras destacadas del pensamiento francés o francófono. Ni más ni menos que Pierre Ryckmans, alias Simon Leys, el sinólogo belga autor en 1971 de Los trajes nuevos del presidente Mao aludió a 
los Escolios gomezdavilianos hablando de sus lecturas de cabecera del momento (invierno 2011) en una entrevista para la revista de la Asociación de estudios chinos de Australia ${ }^{2}$.

Más recientemente dos filósofos franceses de los más mediáticos y polémicos han hecho referencia a Gómez Dávila. Se trata de Alain Finkielkraut en una entrevista al semanal Le Point del año $2015^{3}$ y del filósofo Michel Onfray. Cabe resaltar también dos libros de corte más periodístico con capítulos dedicados a Nicolás Gómez Dávila: Frédéric Schiffter, Le charme des penseurs tristes, Flammarion, publicado en agosto de 2013, y Bruno de Cessole, L'internationale des franc-tireurs, L'Éditeur, en octubre de 2014. Se entiende que queda mucho por hacer para difundir la obra y el pensamiento gomezdaviliano en Francia, a lo cual nos hemos dedicado mal que bien, por ejemplo, en un artículo de la revista Catholica publicado en invierno de 2015: "Nicolás Gómez Dávila y las paradojas del conservadurismo". Por consecuente, la conclusión de esta primera parte de nuestra investigación resulta ser obvia: en Francia, en cuanto a la recepción de la obra gomezdaviliana, todo o casi todo queda por hacerse. Sin embargo, este problema nos conduce a otro, mucho más profundo, que nos gustaría analizar en una segunda parte: ¿por qué no ha habido recepción a nivel académico, o casi, de Gómez Dávila en Francia?

\section{La no recepción de Gómez Dávila en Francia}

Este problema de la no recepción —más que de la recepción- de la obra gomezdaviliana en Francia está relacionado no sola y directamente a una cuestión editorial y/o de traducción como lo hemos visto anteriormente, sino también, a un asunto intelectual y académico que tiene mucho que ver, según nuestra tesis, con la concepción francesa y moderna de la filosofía, y a lo que hemos llamado la "anti-filosofía" Gómez Dávila. Mutatis mutandis puede que esté ocurriendo con "el Nietzsche colombiano" (Quevedo, 1999), lo que ocurrió con él mismo Nietzsche en Francia, para tomar el ejemplo más significativo y cercano tanto en la forma como en el fondo. Traducido desde los años 1890, el filósofo de Sils-Maria ha sido considerado en el país galo como un mero poeta hasta su "rehabilitación" académica llamada por Michael A. Peters "construcción francesa de Nietzsche" (2007, p. vii), gracias a las investigaciones de Michel Foucault,

\footnotetext{
"DS: What are you reading at the moment? PR: Leszek Kolekowski, My Correct Views of Everything; F.W. Mote, China and the Vocation of History in the Twentieth Century A Personal Memoir; and for bedside reading, I keep constantly dipping into two huge collections of sardonic aphorisms (gloriously incorrect!) by two eccentric and lonely geniuses: Cioran's posthumous notebooks (Cahiers) and Nicolás Gómez Dávila's Escolios a un texto implícito (my Spanish is very primitive, but have the help of two volumes of French translations)" (Ryckmas, 2011).

“Quand j'ai un accès d'immodestie, je me récite cette phrase du philosophe colombien Nicolás Gómez Dávila: «L'intellectuel n'oppose pas à l'homme d'Etat l'intégrité del'esprit mais le radicalisme de l'inexpérience»".
} 
Michel Deleuze y Jean-François Lyotard en un primer momento, seguido de Jean Granier y Eric Blondel y más recientemente Patrick Wotling o Dorian Astor. Como bien lo ha mostrado Louis Pinto (1995) en su análisis de la recepción del filósofo alemán en Francia, el mundo universitario francés se había desinteresado de esta llamada "filosofía de amateurs", de un pensamiento tildado de antimoralista y antidemocrático, aristocrático y reaccionario - hasta "nazi" en una época- dejando su lectura e interpretación a los "artistas", escritores o, peor aún, ideólogos políticos cercanos al fascismo o al nacionalismo. Pero más allá de una cuestión política, el problema de la recepción de Nietzsche en Francia nos parece ligado a la influencia del hegelianismo sobre la concepción francesa y universitaria de la filosofía. Del hegelianismo y de su heredero transformado y dogmatizado, el marxismo como canon de la filosofía, es decir: el sistema. Por lo tanto, según esta concepción toda filosofía no sistemática o asistemática no es una filosofía, peor aún, es una anti-filosofía. Cabe resaltar que esta concepción se desarrolla en el país que inventó el "ensayo" y el "aforismo" como estilo filosófico y género literario desde el autor de los Ensayos hasta Emil Cioran, pasando por los moralistas del siglo XVII y algunos filósofos de la Ilustración, Voltaire entre otros.

\subsection{La "anti-filosofía" gomezdaviliana}

Como todo verdadero filósofo, Gómez Dávila se pregunta de manera recurrente, al compás de sus notas y escolios sobre la propia filosofía. Aunque no era filósofo de profesión y si bien no la formula directamente, una cuestión atraviesa su obra, a nuestro modo de ver imprescindible para entenderla: "ipor qué la filosofía?" o más bien "ipara qué la filosofía?". Esta cuestión no es nada anodina aún más si se recuerda esta confidencia final en los últimos Escolios: "He visto la filosofía desvanecerse poco a poco entre mi escepticismo y mi fe" (Gómez Davila, 1992).

Ahora bien, esta cuestión fundamental, inaugural deberíamos escribir — “¿Por qué o para qué la filosofía?" — se encuentra obviamente y consubstancialmente vinculada a esta otra sin la cual no se puede responder: "iqué es filosofía?". La tercera pregunta, continuando lógicamente las dos anteriores es: "¿cómo filosofar?", es decir "¿cómo transmitir y comunicar la filosofía?".

En efecto, Nicolás Gómez Dávila se pregunta constantemente sobre el problema del estilo en filosofía, desarrollando un verdadero arte poético o más bien filosófico de escribir, justificando el uso de la forma breve, defendiendo incluso la filosofía del fragmento. Del mismo modo intenta en numerosas ocasiones responder a la segunda cuestión ("iqué es filosofía?") aunque no siempre de manera positiva, muy lejos de allí. Los pasajes respecto a la filosofía abundan en su obra con un cierto menosprecio, e incluso calumnia, tanto, que se podría ver en él un "antiphilosophe" (anti-filósofo) típico, e incluso caricaturesco, viniendo de su 
herencia contrarrevolucionaria, conservadora o reaccionaria, y de su catolicismo en muchos aspectos intransigente, y hasta fideista para ser más preciso (Urbanek, 2013, pp. 41-49). Entre el escepticismo, hacia la posibilidad de la razón para alcanzar la verdad y la fe en una verdad revelada, queda así muy poco espacio para el ejercicio de la filosofía dado a menudo por inútil, o incluso ridículo, como las citas siguientes, entre otras muchas, lo manifiestan:

- "Llamamos filosofía la lógica del discurso cuando tiene lo absurdo por tema" (Gómez Dávila, 1977, I, 54).

- "El hombre común yerra en la obscuridad, el filósofo se equivoca a la luz del día" (161).

- "[...] Un diploma de dentista es respetable, pero uno de filósofo es grotesco" (201).

- "Escucho toda prédica con involuntaria ironía. Tanto mi religión como mi filosofía se reducen a confiar en Dios" (258).

- "No se debe tomar en serio ninguna filosofía, ni burlarse de ninguna. Si alguna fuese simplemente cómica habría que desesperar del hombre, si alguna fuese totalmente seria habría que desesperar del mundo" (282).

- $\quad$ "El léxico filosófico se divide en palabras para pensar y en palabras para creer que pensamos" (377).

- "La filosofía que no se contenta, a la postre, con catalogar dificultades meramente resulta cómica a la larga" (450).

- "La deducción filosófica es el arte de transformar, una observación exacta, pero limitada, en un sistema comprehensivo, pero falso" (Gómez Dávila, 1977, II, 9).

- "La filosofía, infortunadamente, consiste en un discurso, que si no es coherente no es nada, sobre un universo, que no es nada si es coherente" (138).

- "La filosofía es la parte de la retórica donde orador y auditorio se confunden en una sola persona. Filósofo es el que no adopta sino los argumentos con que se convenció a sí mismo" (209).

- "La «praxis» es el pretexto con que la filosofía dimite" (369).

- "Las filosofías comienzan en filosofía y acaban en retórica" (Gómez Dávila, 1992, 59).

- "Las filosofías no se tornan propiamente obsoletas, se vuelven aburridas" (120).

- "Lo difícil del filósofo difícil suele ser más su lenguaje que su filosofía" (123).

- "Los que se ocupan de filosofía no la tomarían tan a serio, si generalmente no vivieran de profesarla" (130).

- "Toda mitología es en cierta manera cierta, mientras que toda filosofía es en cierta manera falsa" (152). 
"Falsedad", "abstracción", “cobardía", "ligereza", "retórica”, “jerga", "aburrimiento", etc., tenemos aquí casi todo el directorio de los adjetivos infamantes que no habrían rechazado ("ateísmo" aparte) ni un Joseph de Maistre, ni un Edmund Burke, ni un Nicolas de Bonald, entre los más famosos contrarrevolucionarios, para (des)calificar la filosofía y sobre todo los llamados "Philosophes" ("Filósofos") como los nombraban en ese entonces los adversarios de las Luces y como los Enciclopedistas se bautizaron ellos mismos (Masseau, 2000; Mac Mahon, 2001, cap. 1 y 2). La contradicción entre la palabra y los actos, o entre el discurso y la realidad, en suma entre la teoría y la praxis, atraviesa la argumentación antifilosófica como oposición fundamental entre la mentira, la ilusión o el error y la verdad, la cual se reduciría también en términos epistemológicos, a la oposición entre la novedad inexperta y la antigüedad probada (prejuicio, costumbre), entre la Razón abstracta y la Sabiduría práctica, entre idealismo y realismo o pragmatismo; en fin y en léxico político, entre Revolución y Reacción o Tradición tal cual lo podemos encontrar en la obra del Old Whig Burke, Reflections on the Revolution in France [Reflexiones sobre la revolución en Francia]. Pero más concretamente, detrás de estas críticas anti-filosóficas gomezdavilianas, y reaccionarias en general, nos parece también ser criticada aquí la pretensión de la filosofía al "conocimiento absoluto" (absolute Wissen) en vocabulario hegeliano y a la cientificidad perfecta según un modelo positivista, es decir, cientificista y sobre todo enciclopedista. Las incidencias no faltan igualmente en la obra gómezdáviliana de la crítica a la reducción y/o asimilación de filosofía a la ciencia como se entiende a la lectura de estos otros escolios:

- "La desorientación intelectual en que vivimos no proviene del fraccionamiento creciente de la ciencia, sino del rigor creciente de la filosofía. El hombre camina a trompicones cuando la filosofía rehúsa ensuciarse las manos" (Gómez Dávila, 1977, I, 419).

- "El filósofo que adopta nociones científicas predeterminó sus conclusiones" (47).

- "Mientras mayor sea la cantidad de nociones científicas que el filósofo integra a su sistema, más rápidamente el sistema se desploma" (Gómez Dávila, 1977, II, 65).

- "La filosofía que se tecnifica no gana en certidumbre, sino en ininteligibilidad" (Gómez Dávila, 1992, 29).

- "Lo único que no se le debe perdonar al filósofo es que las ciencias lo encandilen y la técnica lo deslumbre" (123).

- "Los métodos de la investigación científica son filosóficamente interesantes, sus conclusiones son filosóficamente indiferentes" (143).

Contrariamente a una idea que se ha vuelto común, la filosofía a pesar de sus ínfulas modernas, no es una ciencia según Gómez Dávila, aunque se le juntó el epíteto de "humana" (contradictio in adjecto) y se extravía a buscar asemejarse 
a ella. Hay que acordarse de los ataques de Pascal contra Descartes "inutile et incertain" ["inútil y dudoso"] (1963, p. 887), de las cargas de Kierkegaard contra la lógica hegeliana como pretendida ciencia filosófica (Migajas filosóficas), de las burlas de Nietzsche al "charlatanismo de forma matemática" (Más allá del bien y del mal, §5) destinadas a la Ética de Spinoza y sus demostraciones more geometrico o también, más próximo, de las resistencias de Wittgenstein a la aplicación del método científico a la filosofía en el Tractatus lógico-philosophicus. De hecho, para este último, el paradigma filosófico no es científico sino literario y su elección del aforismo obedece a un significado filosófico y ético propio como se ha muy bien demostrado (Gabriel, 2001, pp. 111-126), (Gibson and Huemer, 2004, pp. 15-124). Filosofía debería escribirse en poemas (nur Dichten) afirma incluso el filósofo y lógico austríaco en un apunte de sus cuadernos de 1933-1934, insistiendo sobre el proceso de escritura en la filosofía, la composición (Dichtung) más que en la argumentación. Una asimilación que tiene al método de escritura obviamente (pero no solamente), método contrario al modo more geometrico o al constructivismo como paradigma dominante de la modernidad (Brague, 2015; Lachterman, 1989). Se encuentra este mismo paradigma literario en Gómez Dávila que vuelve en varias ocasiones sobre esta asimilación/inspiración, parafraseando a Paul Valéry (1957, I, p. 1256) extraída de su texto sobre Leonardo Da Vinci: "La filosofía es un género literario" (Gómez Dávila, 1977, I, 312). Como para Valéry, como para Wittgenstein, la filosofía según Gómez Dávila debe ser obra literaria, por ejemplo, cuando afirma: "La filosofía se vuelve más sensata cuanto más se aproxima a la literatura. La prosa limpia es el escollo de la especulación extravagante" (Gómez Dávila, 1977, II, 42), O bien: "La literatura es la más sutil, y quizá la única exacta de las filosofías" (157).

La cuestión aquí, se entiende, tiene que ver con el manejo del idioma, con el uso justo e íntegro de la lengua sin que ésta "se pague de palabras" como dice la expresión francesa, es decir, conceda al lenguaje más que debe y que cree o hace creer hasta caer en una tecnicidad —la "jerigonza filosófica" (Gómez Dávila, 1992, 165)_, o la retórica cuyo pecado original reside en una forma de nominalismo moderno considerando que el nombre —el nombramiento deberíamos decirequivaldría a hacer existir la cosa cuando se trata en realidad de una invención, a saber una "ficción gramatical" (Nietzsche, Más allá del bien y del mal, § 17), o, mejor dicho, de una ficción onomástica en sentido casi propio de la palabra griega (nomastikê). Gómez Dávila sabe bien que algunos filósofos se complacen en las invenciones lingüísticas (que bautizan a veces como "conceptos") y que así se creen grandes inventores filosóficos. En este sentido también Gómez Dávila resulta ser radicalmente antiphilosophique si se considera su desconfianza respecto a la lengua, sobre todo a la lengua filosófica calificada de "jerigonza" y a la argumentación filosófica que se destina a la explicación, es decir, en resumen, el discurso filosófico en general: 
A una filosofía que explica prefiero una filosofía que muestra.

La primera disuelve lo concreto, la segunda agudiza mi percepción de lo real.

Las evidencias persisten aquí, sin duda, en su pura contingencia, pero la necesidad última que nos presentan allí, resulta finalmente mera constatación empírica.

Detrás de la evidencia concreta sólo hay otra evidencia más pobre (Gómez Dávila, 1977, I, 393).

Su preferencia por la "mostración" más bien que para la demostración en filosofía certifica aún de un anti-filosofismo, o incluso de un antirracionalismo típico, heredado de la Contra-ilustración, aunque perteneciente también a otra tradición filosófica posterior - la fenomenología- que predicará en otro contexto una vuelta a las "cosas mismas" es decir, al "concreto" contra la abstracción de las representaciones filosóficas.

Es interesante observar que se trata también de uno de los criterios de la "antiphilosophie" que distingue Alain Badiou desde el principio de su Seminario (2004), término al cual el controvertido universitario francés dio un nuevo significado, destinándose en particular a Nietzsche y Wittgenstein —dos pensadores influyentes en el pensamiento de Gómez Dávila, pero que él hace remontar hasta san Agustín, Pascal, Rousseau y Kierkegaard- otros pensadores a los cuales el Colombiano hace frecuentemente referencia. Un nuevo significado del término "antiphilosophie" que se incorpora sin embargo en parte al antiguo, aunque Badiou lo encuentra en primer lugar en Jacques Lacan (Aubry, 2010, pp. 81-94) y que puede aplicarse a toda una tradición filosófica contraria a la que representa el mismísimo Badiou y que podría resultar sino la "verdadera" filosofía quizá, al menos en su sentido originario, es decir histórico, la auténtica o mejor, una "archi-filosofia" (pp. 92-94). Ahora bien, pues, la "antiphilosophie" no sería tan antifilosófica (Badiou utiliza por otra parte el oxímoron de "filosofía antifilosófica"), o incluso más, o mejor. Bajo esta palabra Badiou designa un pensamiento que se opone al discurso filosófico "tradicional" o más exactamente sistemático-racional, precisamente a una construcción de la verdad que no sería de carácter conceptual, académica y sistemática, pero como una búsqueda de orden personal, psíquica o mística. Más exactamente Badiou resalta tres características que conviene destacar:

1. Las declaraciones historiales de la filosofía son absurdas.

2. La esencia de filosofía debe comprenderse en términos de acto más bien que en términos de dispositivos teóricos.

3. El digno acto, no hablador, del pensamiento, que no establece el pensamiento en la confusión entre el pensamiento y el no pensamiento, es el elemento místico, el elemento místico como acto supra-filosófico. Es lo por cual algo se hace ver sin decirse. 
Estas tres características observadas por Badiou corresponden a la concepción gomezdaviliana y "mística" de la filosofía y nos permiten introducir otra interrogación, más esencial seguramente para la filosofía y que permitiría quizá solucionar la anterior, a saber: "¿Qué es filosofía?" y más concretamente si se considera que el filósofo busca la verdad: "¿Qué es la verdad?".

\subsection{Una concepción "epifaníca" de la verdad}

En efecto, si se coloca uno al lado de otro, varios escolios consagrados a la verdad, su definición y sus condiciones de "mostración" más que de su demostración (esquema matemático y en consecuencia deductivo-lógico opuesto al paradigma poético), constatamos esta dimensión concreta, personal, individual e incluso subjetiva de la experiencia de la verdad distinta de su construcción conceptual:

- "La verdad no es, allende las cosas, el esquema de éstas o su fórmula intelectual: verdad es el nombre de la realidad que percibimos en su plenitud de realidad" (Gómez Dávila, 1954, 72).

- "La verdad es persona." (Gómez Dávila, 1977, I, 64), "La verdad no es juicio, sino adhesión a una evidencia concreta" (65).

- "Para que una verdad nos convenza se requiere que una verdad equivalente madure en nuestras almas" (112).

- "Toda verdad va de la carne a la carne" (210).

- "La verdad es una sonoridad peculiar a ciertas voces cuando ciertas evidencias las conmueven. La verdad no yace ni en cualquier evidencia, ni en cualquier voz" (255).

- "La más simple verdad es tan compleja que ninguna formula la expresa, y requiere para expresarse el contexto global de una persona y de una vida" (255).

- "Mi verdad es la suma de lo que soy, no el simple resumen de lo que pienso" (287).

- "Buscar la "verdad fuera del tiempo" es la manera de encontrar la "verdad de nuestro tiempo" "El que busca la "verdad de su tiempo" encuentra los tópicos del día" (Gómez Dávila, 1977, II, 149).

- "No pertenezco a un mundo que perece. Prolongo y transmito una verdad que no muere" (500).

- "La verdad no es separable de la carne individual que hiere, pero difiere de la herida" (Gómez Dávila, 1986, I, 52).

- "Toda verdad nace entre un buey y un asno" (122).

- "La indemostrabilidad de la verdad nos pide estructuras que den la más amplia cabida al error. Jerarquicemos para no recurrir a la violencia" (188).

- “La verdad total no será empacho de un proceso dialéctico que engulle 
todas las verdades parciales, sino límpida estructura en que se ordenan" (79).

- "La verdad convence con un guiño; el error necesita discursos" (Gómez Dávila, 1986, II, 20).

- "La verdad se expresa también con torpeza, pero la belleza de una frase garantiza su verdad" (93).

- "La imposibilidad de demostrar una verdad no implica la imposibilidad de mostrarla. Aún allí donde no hay verdades demostrables puede así haber verdades inter-subjetivas" (95).

- "Más que una proposición impersonal, la verdad es una manera de pensar y de sentir" (Gómez Dávila, 1992, 15).

- "La verdad no es producto de raciocinios, sino postulado indemostrable" (157).

No hay posibilidad según Gómez Dávila, como se mencionó en primer lugar, de demostración de la verdad, pero de una mostración, de la misma manera que según Wittgenstein, lógico contra el método matemático aplicado a la filosofía. Que una verdad sea indemostrable no implica con todo que no sea mostrable. Por lo tanto, y, en segundo lugar, la introducción de ésta se hará según un método épifanico (Abad, 2008), es decir, de una aparición como una "evidencia" y no según el método dialéctico de la explicación o de la argumentación. La metáfora evangélica del buey y el asno tiende a reforzar esta dimensión épifanica, y por definición casi mística, de la verdad, pero incluso sería posible encontrar una posición o mejor dicho una disposición fenomenológica en referencia a Husserl en este planteamiento gomezdaviliano de la verdad como "aparición" y no demostración. En efecto Gómez Dávila no parece tan distante aquí de la definición fenomenológico-existencial de la verdad como "descubrimiento" según su etimología griega a la cual recurría también el autor de Ser y Tiempo. Se podría por otra parte poner esta definición en relación con su determinación de la filosofía como obra literaria u obra poética (Wittgenstein) y la de Heidegger del arte como Poema, es decir, aparición o instauración de la verdad. Consecuencia de esta mostración de la verdad, su carácter personal, encarnada en una persona, una voz, una manera de sentir y de pensar (una stimmung diría el alemán) de un individuo. Que no hay demostración no significa, al contrario, que no deba haber apropiación de la verdad, sino una apropiación personal fruta de una especie de maduración, casi vegetal. La evidencia surge en absoluto, pero no donde quiera, ni en cualquier momento ni de todos modos. Sin embargo, en tercer lugar, lo que precede no significa que habría una relatividad y una parcialidad de la verdad. Gómez Dávila desafía también el relativismo contemporáneo planteando lo que se podría nombrar una transcendencia de la verdad. Transcendencia que supone por supuesto su permanencia y su intemporalidad. Se ve bien luego cómo se inscribe en una corriente "antiphilosophique" no solamente en su 
sentido contrarrevolucionario o conservador, pero, según el análisis mismo de Alain Badiou y hasta en la preocupación por la forma literaria, el estilo, del que dependen la transmisión de la verdad, preocupación que se encuentra en estos otros "antiphilosophes" que son Pascal, Kierkegaard, Nietzsche, Wittgenstein. Nada asombroso pues que el colombiano se sitúa de este modo en una filiación filosófica de "aficionados" más que de "profesionales" de la filosofía: "El gremio de filósofos profesionales sufre de inanición filosófica si no ingurgita, de vez en cuando, un aficionado: Sócrates, Descartes, Hume, Kierkegaard, Nietzsche" (Gómez Dávila, 1977, II, 34).

Gómez Dávila distingue claramente dos corrientes en la historia de filosofía, la "profesional", que se podrá calificar de "escolástica" en sentido general que consistirá en un discurso filosófico sobre filosofía, una teoría; y, aquélla, "amatrice" o "aficionada" en el sentido que es ejercida por pasión y no por especialización, que se podrá también calificar de "cósmica" (mundana) o "existencial" consistiendo en una elección de la vida filosófica, una ética y una práctica en el linaje socrático y antiguo tal como Pierre Hadot lo explicó a lo largo de toda su obra. Gómez Dávila, de una manera más metafórica, va a separar dos campos en la historia de la filosofía: el de los "usurpadores" y el de los "colonizadores" según su propia metáfora (Gómez Dávila, 1977, II, 25), es decir, al fin y al cabo, entre los que se niegan y los que aceptan construir un sistema filosófico: "Como la filosofía es tierra colonizada por aristotélicos y kantianos, un Kierkegaard o un Nietzsche, más que soberanos constitucionales de sus reinos, parecen usurpadores imperiales" (25).

Pero estos "usurpadores" geniales se inscriben sin embargo en una tradición originalmente o "antiguamente" filosófica según otros (Pierre Hadot en primer lugar) para los cuales la filosofía es ante todo una actividad, una actividad es decir un arte en sentido propio, de vivir, pensar, leer y escribir (porque también scribere es agere): arte que empieza como un arte de cuestionar, plantear problemas y no dar respuestas o "soluciones".

En esta medida el colombiano, como sus ilustres antecesores, desdeña toda sistematización filosófica - "O se pertenece a la posteridad de Hegel, o se pertenece a la posteridad de Schopenhauer. Tertium non datur" (Gómez Dávila, 1977, II, 183)—; desafía toda institucionalización filosófica — “Lo religioso, lo militar, lo político, se institucionalizan. Pero ni la filosofía, ni las artes, ni las letras.", (255)—; y, por ende, desprecia toda profesionalización filosófica: "Filósofo honesto es el que no deja que su oficio piense por él" (Gómez Dávila, 1992, 32).

Tres peculiaridades que lo alejan de une cierta concepción francesa y académica de la filosofía, más no de lo que sería la condición autentica de la filosofía, exactamente "zetética" en el sentido en el cual el filósofo austro- 
americano Eric Voegelin interpreta la palabra griega: "The philosophers' truth does not become a possession; it remains the truth of the search (zetesis) in erotic tension toward the mysterious ground of existence" (Voegelin, 2000, p. 236).

Esta idea "zetética" y antiacadémica de la filosofía evita por lo tanto caer en este defecto indudablemente y profundamente anti-filosófico, en el sentido fuerte y verdadero de la palabra, que consiste a buscar respuestas, o un "sistema", antes de formular preguntas, es decir una concepción ideológica: "Las «soluciones»son las ideologías de la estupidez" (Gómez Dávila, 1977, II, 89).

\subsection{Zetética filosófica us. dogmatismo filosófico}

En efecto, la ideología resulta ser exactamente lo contrario de la filosofía concebida en este sentido originario en la medida en que rechaza e incluso niega el cuestionamiento de miedo a ver hundirse el sistema. La ideología o el dogmatismo filosófico, la "dogmatomaquia ideológica" para reanudar exactamente la expresión de Voegelin que se basa en "la denegación, elevada a la fila de sistema, de la apercepción de la realidad —- "Apperzeptionsverweigerung" (Voegelin, 2011, p. 90)—, desembocando en una prohibición de la cuestión que el filósofo austro-americano encuentra formulada en Auguste Comte y Karl Marx, pero que no le es propia a ellos, sino que ellos la formularon explícitamente. Así pues, cuando el primero rechazaba las "cuestiones ociosas", el segundo las proscribía sencillamente: "Denke nicht, frage mich nicht." ["No piensa, no plantea cuestiones"], (Voegelin, 2011, p. 90; 1999, p. 263). Una táctica que Voegelin encontraba en los hegelianos pero que resulta típica de todos los ideólogos y hacedores de sistema, que él no duda en calificar de estafadores intelectuales. La consecuencia de esta estafa consiste en querer substituir la "segunda realidad" - esta imagen falsa de la realidad que suministra el sistema — a la primera. Ahora bien, esta mentira que funciona como una "segunda realidad", identificada a la ideología, se basa también en la lengua, pero una lengua destruida y dogmática, un idioma ficticio que tiene una existencia propia sin relación con la (primera) realidad, método que hemos llamado como ficción onomástica. Por esta razón estamos en condiciones de concluir que la ideología, y su puesta en forma como "sistema" en el dogmatismo filosófico o pseudo-filosófico, es la verdadera -y la más peligrosa-anti-filosofía. Resulta incluso peor que anti-filosófico, es el síntoma y el sistema del "no pensamiento" como lo expresará el mismo Gómez Dávila a su manera: "Las ideologías se inventaron para que pueda opinar el que no piensa" (1977, II, 227).

Al contrario, la filosofía en su índole "zetético", es decir socrático, consiste en un cuestionamiento permanente, una erótica del espíritu, una inquietud intelectual, o incluso espiritual, que permite nunca perder pie con la realidad, 
la primera, es decir con la experiencia humana abierta a la transcendencia. Por eso a la cuestión "Why Philosophize?, Voegelin podía responder: "To Recapture Reality!" [“¿Por qué filosofar? iPara reencontrar la realidad!"] (2011, cap. 22).

En su crítica del sistema filosófico, en su concepción de la filosofía como un modo de vida, en su búsqueda de un estilo adaptado al discurso filosófico, Gómez Dávila se incorpora a esta preocupación voegeliniana de una restauración del nivel de cuestionamiento platónico-aristotélico, o digámoslo más generalmente, de una vuelta a la fuente socrática de la filosofía ("zetética"). Responder a la cuestión "¿qué es filosofía?" supone pues volver de nuevo a la actitud filosófica inaugural, la de Sócrates: el cuestionamiento. "Filosofía socrática significa: cuestionar" recordaba por su parte también el filósofo Leo Strauss (1997, pp. 393-436). Es también la posición gomezdaviliana frente al "oficio" de filosofar: "La filosofía es el arte de formular lúcidamente problemas. Inventar soluciones no es ocupación de inteligencias serias" (Gómez Dávila, 1977, II, 54).

Este método zetético constituye incluso la diferencia fundamental entre las ciencias y la filosofía y explica por qué la segunda no puede reducirse a las primeras pese al proyecto moderno de constituir ciencias dichas "humanas" —en lugar de Studia humanitatis - sobre el modelo de las ciencias naturales o físiconaturales: "Lo grave de las ciencias naturales sería que se perdieran las respuestas, en filosofía que se olvidaran las preguntas" (Gómez Dávila, 1977, I, 410). E incluso cuando se encuentran respuestas en filosofía aquellas sólo constituyen el inicio de otros problemas en una cadena continua e ininterrumpida: "Las soluciones en filosofía son el disfraz de nuevos problemas." (Gómez Dávila, 1992, 25). O, en el mismo sentido: "El acto filosófico genuino está en descubrir un problema en cada solución" (Gómez Dávila, 1977, I, 146). Pero este método gomezdaviliano resulta también trascendental para entender la historia misma de la filosofía en la medida en que cada respuesta filosófica a un problema permanece como otra tentativa de responder a una interrogación que la funde y que por lo tanto la vuelve inteligible: "Cualquier filósofo es indescifrable a quienes averiguan qué contesta antes de saber qué pregunta" (Gómez Dávila, 1977, I, 24).

\section{Conclusión}

Por lo tanto, sería de esperar en Francia una reevaluación académica del pensamiento gomezdaviliano dentro de una redefinición no ideológica y no sistemática de la filosofía. Es preciso agregar que la una no podrá ocurrir sin la otra, o la primera, tal vez, podría conllevar a la secunda solamente si se retoma el rumbo socrático-zetético del quehacer filosófico, y, en particular, su definición como forma de vida tal como el historiador de la filosofía Pierre Hadot lo ha ido haciendo a lo largo de su obra, especialmente en lo concerniente a la filosofía 
antigua. Precisamente Van Delft ha demostrado en cuanto a los moralistas que bajo su exigencia "casi ascética" (2008, p. 343), este estilo típicamente francés pertenece a la tradición antigua no solamente en su forma sino en su fondo.

Hay en el moralista europeo, y francés en especial, un magister vitae, un director espiritual y en la escritura de aforismos un verdadero ejercicio espiritual (Van Delft, 2008, p. 82). Es esta Idea escondida y verdadera de la filosofía para retomar la expresión de Kant (1980, p. 8 y 12), que encontramos en los Ensayos de Montaigne, en los Pensamientos de Pascal, en las máximas de La Rochefoucauld, los aforismos de Vauvenargues, Chamfort, Rivarol, Voltaire y, en el siglo XX, los de Valéry, Cioran, Thibon, entre otros; Idea de la filosofía ligada a una forma breve y discontinua, un "estilo cortante y corto" (Van Delft, 2005, p. 132), proseguidos por el Colombiano de espíritu francés hasta en su ironía volteriana.

En verdad, no deja de ser sorprendente la no o escasa recepción de su obra en Francia al no ser que aquella estuviere ligada, más que a un retorno de una supuesta y fantaseada "antiphilosophie", a un renacer del "esprit français" [espíritu francés] y de los dos rasgos de la filosofía francesa según Bergson: el espíritu antisistemático y el sentido moral o psicológico (Bergson, 1915).

\section{Referencias}

Abad Torres, A. (2008). Pensar lo implícito: en torno a Gómez Dávila. Pereira: Centro de Recursos informáticos y Educativos-CRIE.

Aubry, G. (2010). La philosophie comme manière de vivre et I'antiphilosophie. En Davidson, A. y F. Worms (Ed.), Pierre Hadot. L'enseignement des antiques, I'enseignement des modernes. Paris: Editions rue d'Ulm.

Badiou, A. (2004). L'antiphilosophie de Wittgenstein. Caen: Nous.

Badiou, A. (2013). Le Séminaire. Lacan, L'antiphilosophie 3 (1994-1995). Paris: Fayard.

Badiou, A. (2015). Le Séminaire. Nietzsche. L'antiphilosophie 1 (1992-1993). Paris: Fayard.

Bergson, H. (1915). La philosophie française, La Revue de Paris, (1915), 236-256.

Brague, R. (2015) Le règne de I'homme. Paris: Gallimard. 
Gabriel, G. (2001) La logique comme littérature ? Remarques sur la signification de la forme littéraire chez Wittgenstein. En Pierre Hadot. (Ed.), Wittgenstein et les limites du langage. Paris: Vrin.

Gibson J. y Huemer, W. (2004). The literary Wittgenstein. New York: Routledge \& Taylor.

Gómez Dávila, N. (1954). Notas. Ciudad de México: El autor.

Gómez Dávila, N. (1977). Escolios a un texto implícito, I y II. Bogotá: Instituto Colombiano de Cultura.

Gómez Dávila, N. (1986). Nuevos escolios a un texto implícito, I y II. Bogotá: Procultura.

Gómez Dávila, N. (1992). Sucesivos escolios a un texto implícito. Bogotá: Instituto Caro y Cuervo.

Kant, I. (1980). Vorlesungen über die philosophische Encyclopädie, dans Kants gesammelte Schriften, XXIX. Berlin: Akademie.

Lachterman, D. (1989). The Ethics of Geometry: A Genealogy of Modernity. New York: Routledge.

Mac Mahon, D. (2001). Enemies of the Enlightenment, the French CounterEnlightenment and the Making of Modernity. Oxford: Oxford University Press.

Masseau, D. (2000). Les Ennemis des philosophes : I'antiphilosophie au temps des Lumières. Paris: Albin Michel.

Nietzsche, F. (2012). Más allá del bien y del mal (A. Sánchez Pascual, Trad.). Madrid: Alianza.

Pascal (1963). Pensées. Paris: Le Seuil.

Peters Michael, A. (2007). Prefacio a Fitzsimons Peter. En Nietzsche, Ethics and Education. Roterdam: Sense Publishers.

Pinto, L. (1995). Les Neveux de Zarathoustra. La réception de Nietzsche en France. Paris: Le Seuil.

Quevedo, A. (1999) ¿Metafísica aquí? Ideas y Valores, (111), 79-88. 
Ryckmas, P. (2011). Interview Professer Pierre Riyckmas. Chinese Studies Association of Australia Newsletter, 41, 10-12. Recuperado de http://www.csaa. org.au/wp-content/uploads/2014/04/CSAA_Newsletter_41.pdf

Strauss, L. (1997). Cohen und Maimuni. En Meier (Ed.), Gesammelte Schriften Band 2 Philosophie und Gesetz-Frühe Schriften. Stuttgart: Metzler.

Urbanek, K. (2013). La recepción de la obra de Nicolás Gómez Dávila en Polonia, Pensamiento y Cultura, 16(2), 33-49.

Valery, P. (1957). OEuvres, t. I. Paris: La Pléiade/Gallimard.

Van Delft, L. (2005). Les spectateurs de la vie. Généalogie du regard moraliste. Saint Nicolas: Presses de I'Université de Laval.

Van Delft, L. (2008). Les moralistes. Une apologie. Paris: Gallimard Folio-Essais.

Voegelin, E. (1999). Science, Politics, and Gnosticism, Modernity without restraint, Collected Works of Eric Voegelin, (5). Columbia-London: University of Missouri Press.

Voegelin, E. (2011). Autobiographical reflections. London: University of Missouri Press. 